\title{
Clinical Study on Pulmonary Hypertension Caused by Genetic Immunity and Environment
}

\author{
WANG GU XIAN ${ }^{1, a}$, QIN QING YING ${ }^{1}$, ZHA YAN JING $^{1}$, HE WEN YUAN ${ }^{1 *}$ \\ ${ }^{1}$ Company Name: QUJING MEDICAL COLLEGE \\ Corresponding author: HE WEN YUAN
}

\begin{abstract}
Pulmonary hypertension is a chronic pulmonary vascular disease. In recent years, quite a number of studies suggest that the increase of pulmonary artery pressure is the result of multiple factors. A better understanding of the related factors is of great significance to the prevention, diagnosis, treatment and prognosis of pulmonary hypertension. Based on this, this paper studies the genetic immunity and environmental induced pulmonary hypertension. In this paper, 60 patients with pulmonary hypertension in our hospital from January 2020 to the first half of July 2020 were selected as the research objects. According to the pathogenic factors, the patients were divided into groups. Among them, pulmonary hypertension caused by genetic factors was group A, and pulmonary hypertension caused by environmental factors was group B. This study found that the incidence rate of family genetic history reached $6 \%$, and the proportion of diseases caused by immune factors accounted for $2 \%$. Because of the high altitude hypoxia environment, about $5 \%$ accounted for $5 \%$. Smoking is the main factor leading to the morbidity of pulmonary hypertension, accounting for up to $70 \%$. The results of this study provide theoretical basis for the treatment of pulmonary hypertension caused by genetic immunity and environment.
\end{abstract}

\section{Introduction}

Pulmonary hypertension is a malignant progressive disease [1-2]. The main clinical manifestation of pulmonary hypertension is the gradual increase of right ventricular afterload, which leads to right heart failure and even death [3-4]. The incidence rate of pulmonary hypertension is increasing year by year. More and more people begin to realize the harm of pulmonary hypertension and its complications. Meanwhile, more and more studies are also being carried out on the pathogenesis of pulmonary hypertension. People expect to intervene in the development of pulmonary hypertension early, and provide a reference for the treatment of pulmonary hypertension. [5-6]

Relevant studies have pointed out that the influencing factors of pathogenesis of pulmonary hypertension include hypoxia, genetic immunity, congenital, smoking and so on. Therefore, the treatment of pulmonary hypertension should also start from the above factors [78]. In recent years, the research on pulmonary hypertension in China has focused on the pathogenesis and diagnosis and treatment, and made significant progress [9-10]. Understanding the pathogenesis and pathogenesis of pulmonary hypertension is conducive to targeted treatment. It is of great significance for the clinical treatment of pulmonary hypertension to explore the genetic immunity and environmental induced pulmonary hypertension.
In this paper, the definition of pulmonary hypertension is described, and the treatment methods of pulmonary hypertension are introduced. We know that in the traditional drug treatment, the effect of drug intervention is mainly concentrated in the patients with pulmonary hypertension who respond to the acute drug experiment, so the effect is not ideal, while the effect of prostacyclin treatment and endothelin receptor antagonist pulmonary hypertension is relatively good. In addition, this article also introduces the pathogenesis of pulmonary hypertension. Finally, through the experimental study of 60 patients with pulmonary hypertension in our hospital from January to the first half of July 2020, we understand the main causes of pulmonary hypertension and the changes of physiological indicators after the onset, so as to provide theoretical data for the follow-up treatment.

\section{Pulmonary Hypertension and Its Treatment}

\subsection{Pulmonary Hypertension}

Pulmonary hypertension (PAH) is a hemodynamic abnormality in which the pressure of pulmonary artery exceeds a certain threshold, which leads to the increase of right ventricular load and right ventricular dysfunction, which leads to a series of clinical manifestations. The diagnostic criteria of hemodynamics were as follows: mean pulmonary artery pressure measured by right heart 
catheterization at sea level and resting state $\geq 25 \mathrm{mmhg}$ $(1 \mathrm{mmhg}=0.133 \mathrm{kpa})$. Pulmonary hypertension is an abnormal hemodynamic state, which is not an independent disease, but includes a variety of clinical conditions.

(1) Classification of pulmonary hypertension

1) Idiopathic pulmonary hypertension is a kind of pulmonary hypertension with unknown etiology. The pathological changes of lung tissue are characterized by endothelial cell swelling, proliferation, smooth cell abnormal proliferation and extracellular matrix accumulation, and the vascular cavity is gradually occluded.

2) Familial pulmonary hypertension is a kind of pulmonary hypertension associated with genetic factors, accounting for $6 \%-10 \%$ of the patients with pulmonary hypertension. Among these patients, $50 \%-90 \%$ of them have found BMPR2 gene mutation. The abnormality of BMPR2 gene is the characteristic manifestation of this kind of patients. Genetic inheritance can advance the onset age of the offspring of patients with pulmonary hypertension, It causes the disease to develop more rapidly.

3) Pulmonary hypertension caused by risk factors or related diseases

4) Persistent pulmonary hypertension in neonates

\subsection{Treatment of Pulmonary Hypertension}

(1) Traditional treatment

In the traditional clinical research, the diagnosis and treatment of pulmonary hypertension are mostly anticoagulant, diuretic, oxygen therapy, including digoxin, calcium channel blockers and so on. But the traditional treatment method is not ideal, the main reason is: the above drug intervention effect is mainly concentrated in patients with pulmonary hypertension who respond to the acute drug experiment.

(2) Prostacyclin in the treatment of pulmonary hypertension

The main mechanism of prostacyclin in the treatment of pulmonary hypertension is to promote the level of adenylate cyclase activation in the receptor, and promote the proliferation of vascular smooth muscle, so as to resist the platelet aggregation reaction in patients with pulmonary hypertension. The earliest intravenous prostacyclin drug used in clinic is eporostrol. Under the intervention of this drug, it can promote the improvement of hemodynamic level of patients, and the effect of prolonging the survival rate of receptor is also significant.

(3) Endothelin receptor antagonist in the treatment of pulmonary hypertension

Since the plasma endothelin level in patients with pulmonary hypertension has an obvious increasing trend compared with healthy controls, the key idea from the perspective of clinical treatment of pulmonary hypertension symptoms is to block the endothelin receptor and reliably inhibit the expression of endothelin activity.

\section{Research Object and Diagnostic Criteria}

(1) Selection of experimental objects
In this paper, 60 patients with pulmonary hypertension in our hospital from January 2020 to the first half of July 2020 were selected as the research objects. The causes of 60 patients with pulmonary hypertension were statistically analyzed. According to the pathogenic factors, the patients with pulmonary hypertension caused by genetic factors were called group A, and those caused by environmental factors were called group B In this paper, the average value of each detection index was used as the experimental data.

(2) Diagnostic criteria of PAH

Under the guidance of color Doppler, pulmonary artery systolic pressure was estimated by simplifying Bernoulli equation

$$
P A S H=4 * V 2+A
$$

$\mathrm{V}$ represents peak velocity of tricuspid regurgitation, a represents right atrial pressure, and $\mathrm{PAH}$ is defined as PASP $\geq 5.33 \mathrm{kpa}$.

(3) Statistical treatment

In this paper, SPSS17.0 software is used for data analysis. The homogeneity and normality of variance were tested. The experimental data of measurement data were expressed as $\bar{X} \pm S$

Let $X_{1}, X_{2}, \ldots X_{n}$ denote from the population sample

$$
m_{i}=\frac{1}{n} \sum_{j=1}^{n}\left(X_{j}-X\right)^{i}
$$

$\mathrm{X}$ is the sample mean.

\section{Clinical Study on Pulmonary Hypertension Caused by Genetic Immunity and Environment}

\subsection{Pathogenesis of Pulmonary Hypertension}

(1) Dysfunction of pulmonary vascular endothelial cells

The imbalance of synthesis and release of vasoactive substances in pulmonary vascular endothelial cells leads to pulmonary vascular lumen stenosis, blockage, vascular contraction, proliferation and hypertrophy of smooth muscle cells and adventitia, which induces and aggravates the occurrence and development of PAH. As a vasodilator, NO plays an important role in relaxing blood vessels, inhibiting the proliferation of smooth muscle cells, and participating in the regulation of vascular pressure and structure. In PAH, endothelial function was impaired, eNOS expression was decreased, no secretion was decreased, and ET-1 secretion led to vasoconstriction and remodeling.

(2) Gene mutation

Bone morphogenetic protein type II receptor (BMPR2) gene belongs to transforming growth factor beta (TGF - $\beta$ ) and belongs to transforming growth receptor superfamily. Its mutation affects BMP signaling, which plays a protective role in pulmonary vessels and induces vascular remodeling. Nitric oxide synthase (NOS) is responsible for the synthesis of No. NOS3 is a key enzyme in the 
physiological regulation of pulmonary smooth muscle cells and vascular endothelial cells. The deficiency of NOS3 gene can lead to protein kinase G tyrosinization and activity damage, and induce pulmonary hypertension. Prostacyclin synthase (PGI2) is the terminal enzyme in the pathway of prostaglandin (PGI2). PGIS deficiency exists in pulmonary capillaries of patients with severe PAH. It has been pointed out that the decrease of PGIS gene expression is related to the disorder of pulmonary vascular endothelial function in patients with severe PAH. $\mathrm{PAH}$

(3) Effects of immune and inflammatory responses on

Studies have shown that inflammatory cells around the reconstructed pulmonary artery infiltrate obviously. These inflammatory cells release a large number of inflammatory factors such as TNF - $\beta$ and IL-6, which lead to injury of pulmonary vascular endothelial cells and proliferation of smooth muscle cells. At present, it has been recognized that inflammation is involved in the occurrence and progress of PAH.

(4) Vascular remodeling

Pulmonary vascular remodeling is an important marker of PAH, characterized by thickening of vascular wall, abnormal proliferation of one or more cells and changes of lung matrix. Studies have shown that the abnormal proliferation of PASMC is involved in pulmonary vascular remodeling, which is characterized by excessive proliferation and hypertrophy of the smooth muscle layer of the media. In addition, a variety of changes in lung matrix components resulted in wall remodeling. The imbalance of matrix protein synthesis and decomposition leads to its occurrence. In addition, hyaluronic acid, as an important component of cell matrix, is an important factor in pulmonary vascular remodeling of IPAH. Matrix metalloproteinases (MMPs) are collagen hydrolases. The imbalance of MMP and tissue inhibitor of metalloproteinase (TIMP) metabolism leads to matrix component accumulation and vascular remodeling.

(5) Thrombus in situ

Platelets, endothelial cells and coagulation chain reaction are involved in its occurrence. The important link of this mechanism is endothelial damage, exposure of collagen fibers under the endothelium, platelet adhesion to collagen fibers. Platelets release platelet activating factor, thromboxane (TXA2) and 5-hydroxytryptamine (5-HT) to accelerate platelet aggregation and form thrombosis.

(6) Ion channel

The change of ion channel may be one of the pathogenesis of PAH. It is found that the abnormal function of calcium channel and potassium channel is involved in the occurrence of PAH.

\subsection{Proportion Analysis of Pulmonary Hypertension Caused by Genetic Immunity and Environment}

In this paper, 60 patients with pulmonary hypertension were classified according to the causes of disease, and the main causes were analyzed. The results are shown in Figure 1.

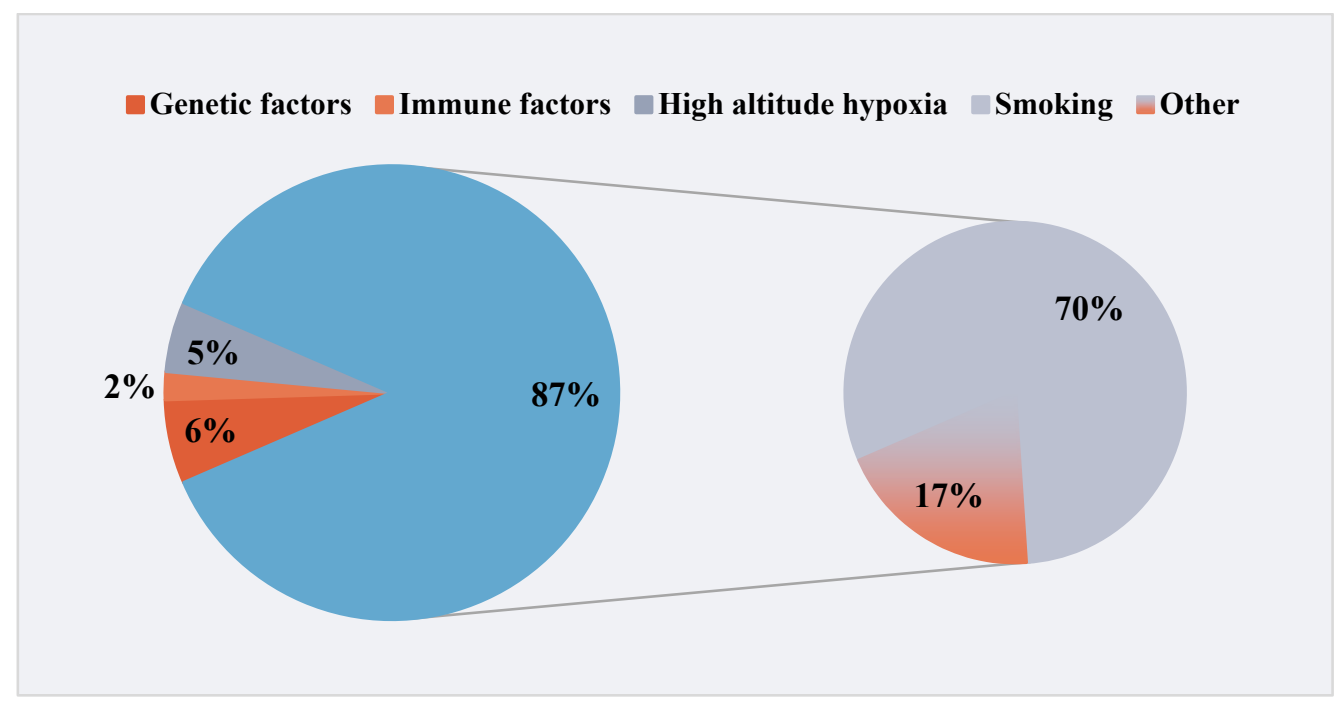

Fig1. Proportion of patients with pulmonary hypertension caused by genetic immunity and environment

The incidence rate of family history is $6 \%$, which is estimated from Figure 1. The proportion of diseases caused by immune factors is $2 \%$, because $5 \%$ of the environment is caused by high altitude hypoxia. Smoking is the main factor leading to the morbidity of pulmonary hypertension. As everyone knows, smoking is harmful to health, and lung and cardiovascular related diseases are closely related to smoking. Current studies have found that more and more diseases are related to smoking, and about $70 \%$ of patients with pulmonary hypertension are caused by smoking.

\subsection{Comparative Analysis of Pulmonary Hypertension Detection Indexes Between Genetic Immune Group and Environmental Factors Group}

In order to analyze the changes of physiological indexes in patients with pulmonary hypertension caused by 
different pathogenic factors, white blood cells, hemoglobin, platelet, creatinine, uric acid and albumin were detected in the two groups. The detection indexes are shown in Table 1 and Figure 2.

Table1. Detection indexes of pulmonary hypertension in genetic immunity group and environmental factors group $(\bar{X} \pm S)$

\begin{tabular}{|l|c|c|c|c|c|c|}
\hline Group & $\begin{array}{c}\text { White blood } \\
\text { cell }\left(* 10^{9} / \mathrm{L}\right)\end{array}$ & $\begin{array}{c}\text { Hemoglobin } \\
(\mathrm{g} / \mathrm{L})\end{array}$ & $\begin{array}{c}\text { Platelet } \\
\left(* 10^{9} / \mathrm{L}\right)\end{array}$ & $\begin{array}{c}\text { Creatinine } \\
(\mu \mathrm{mol} / \mathrm{L})\end{array}$ & $\begin{array}{c}\text { Uric acid } \\
(\mu \mathrm{mol} / \mathrm{L})\end{array}$ & $\begin{array}{c}\text { Albumin } \\
(\mathrm{g} / \mathrm{L})\end{array}$ \\
\hline Group A & $4.7 \pm 0.71$ & $112.3 \pm 2.51$ & $175.3 \pm 5.51$ & $51.2 \pm 1.14$ & $265.1 \pm 5.31$ & $34.6 \pm 1.97$ \\
\hline Group B & $5.3 \pm 1.23$ & $119.4 \pm 1.74$ & $187.6 \pm 6.53$ & $54.5 \pm 1.35$ & $291.9 \pm 3.44$ & $39.8 \pm 2.64$ \\
\hline
\end{tabular}

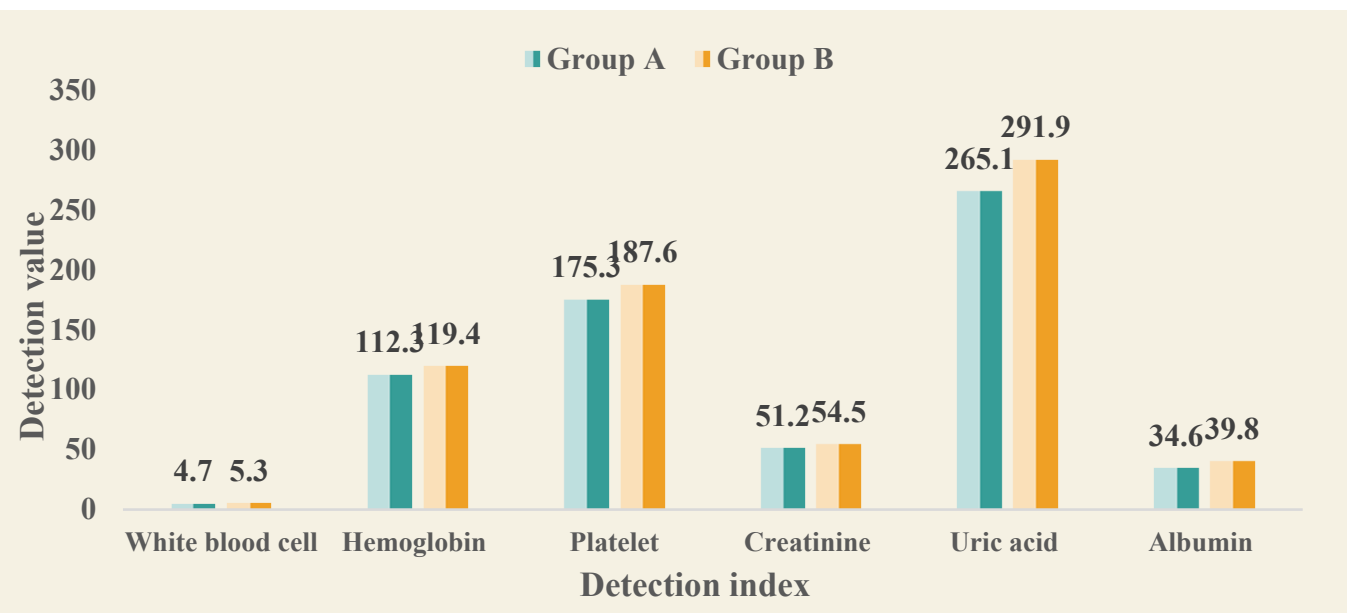

Fig2. Detection indexes of pulmonary hypertension in genetic immune group and environmental factors group

It can be seen from Table 1 and Figure 2 that the detection indexes of patients in group A are generally lower than those of patients in group B. from the data point of view, white blood cell detection value of group A is 4.7 $\pm 0.71\left(* 10^{9} / \mathrm{L}\right)$, hemoglobin is $112.3 \pm 2.51(\mathrm{~g} / \mathrm{L})$, platelet is $175.3 \pm 5.51\left(* 10^{9} / \mathrm{L}\right)$, creatinine is $51.2 \pm 1.14$ ( $\mu \mathrm{mol} / \mathrm{L})$, uric acid is $265.1 \pm 5.31(\mu \mathrm{mol} / \mathrm{L})$, albumin is $4.6 \pm 1.97(\mathrm{~g} / \mathrm{L})$. In group $\mathrm{B}$, WBC was $5.3 \pm 1.23$ (* $\left.10^{9} / \mathrm{L}\right)$, hemoglobin was $119.4 \pm 1.74(\mathrm{~g} / \mathrm{L})$, platelet was $187.6 \pm 6.53\left(* 10^{9} / \mathrm{L}\right)$, creatinine was $54.5 \pm 1.35(\mu \mathrm{mol}$ / L), uric acid was $291.9 \pm 3.44(\mu \mathrm{mol} / \mathrm{L})$, albumin was $39.8 \pm 2.64(\mathrm{~g} / \mathrm{L})$. The results showed that the severity of pulmonary hypertension caused by environment, especially smoking, was higher than that caused by genetic immune factors.

\section{Conclusions}

In recent years, the number of patients with pulmonary hypertension in China has increased year by year. The morbidity and mortality of pulmonary hypertension are very high, which has caused irreparable loss to individuals and society. Understanding the causes of pulmonary hypertension is more conducive to targeted treatment. Therefore, this paper studies the genetic immunity and environmental pulmonary hypertension. It is found that there are many causes of pulmonary hypertension. Smoking is the main cause of pulmonary hypertension, and genetic immunity is also one of the causes. For pulmonary hypertension, its treatment should not only combine with medical treatment, but also eliminate the pathogenesis from the source.

\section{References}

1. Guazzi M, Naeije R . Pulmonary Hypertension in Heart Failure: Pathophysiology, Pathobiology, and EmergingClinicalPerspectives[J]. Journal of the American College of Cardiology, 2017, 69(13):17181734.

2. Dawes T J W , Marvao A D , Shi W, et al. Machine Learning of Three-dimensional Right Ventricular Motion Enables Outcome Prediction in Pulmonary Hypertension: A Cardiac MR Imaging Study[J]. Radiology, 2017, 283(2):161315.

3. Al-Ghanem G, Shah $\mathrm{P}$, Thomas $\mathrm{S}$, et al. Bronchopulmonary dysplasia and pulmonary hypertension: a meta-analysis[J]. Journal of Perinatology Official Journal of the California Perinatal Association, 2017, 37(4):414.

4. Jin $\mathrm{P}, \mathrm{Gu} \mathrm{W}$, Lai $\mathrm{Y}$, et al. The Circulating MicroRNA-206 Level Predicts the Severity of Pulmonary Hypertension in Patients with Left Heart Diseases[J]. Cellular Physiology \& Biochemistry International Journal of Experimental Cellular Physiology Biochemistry \& Pharmacology, 2017, 41(6):2150.

5. Rogers N M, Sharifi-Sanjani M, Yao M, et al. TSP1CD47 signaling is upregulated in clinical pulmonary hypertension and contributes to pulmonary arterial vasculopathy and dysfunction[J]. Cardiovascular Research, 2017, 113(1):15.

6. Driessen M M P, Schings M A, Sieswerda G T, et al. Tricuspid flow and regurgitation in congenital heart disease and pulmonary hypertension: comparison of 
4D flow cardiovascular magnetic resonance and echocardiography[J]. J Cardiovasc Magn Reson, 2018, 20(1):5.

7. Baughman R P , Shlobin O A . Treatment of sarcoidosis-associated pulmonary hypertension: so close, andyet so far[J]. European Respiratory Journal, 2017, 50(4):1701725.

8. Lang I, Meyer B C, Ogo T, et al. Balloon pulmonary angioplasty in chronic thromboembolic pulmonary hypertension.[J]. European Respiratory Review, 2017, 26(143):160119.

9. Vonk Noordegraaf A, Westerhof B E, Westerhof N . The Relationship Between the Right Ventricle and its Load in Pulmonary Hypertension[J]. Journal of the American College of Cardiology, 2017, 69( 2):236243.

10. Augustine D X, Coates-Bradshaw L D, James W, et al. Echocardiographic assessment of pulmonary hypertension: a guideline protocol from the British Society of Echocardiography [J]. Echo Research \& Practice, 2018, 5(3): G11-G24. 